
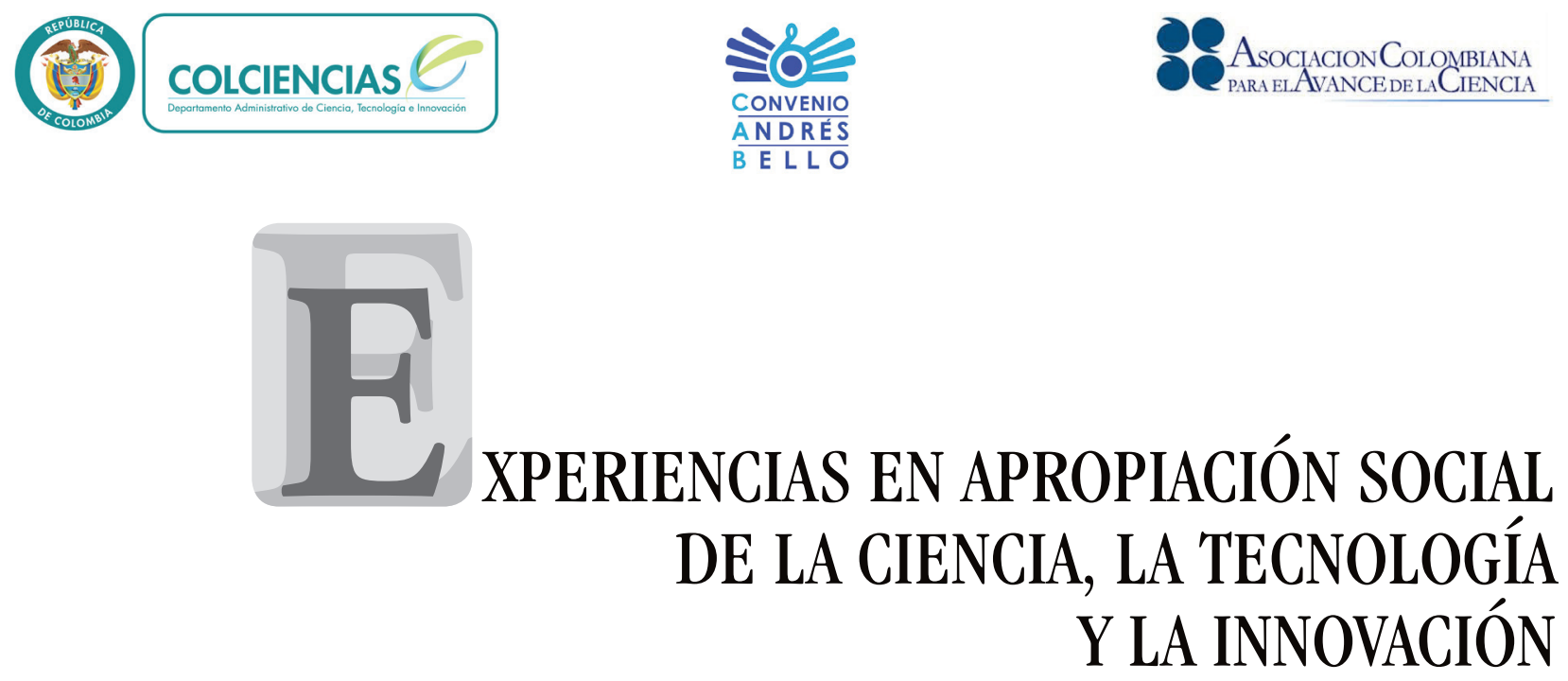

\title{
V Foro Nacional de Apropiación Social de la Ciencia, la Tecnología y la Innovación
}

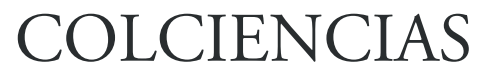

Diana Rocío Rodríguez - Coordinadora de Experiencias

\section{INTRODUCCIÓN}

Como uno de sus principales objetivos, el $V$ Foro Nacional de Apropiación Social de la Ciencia, la Tecnología y la Innovación convocó experiencias de apropiación social de todo el país, para ser presentadas y compartidas durante las distintas actividades desarrolladas a lo largo del proceso, y así, generar comprensiones acerca de cómo se están generando procesos de apropiación de la Ciencia, la Tecnología y la Innovación en los territorios y caracterizar lecciones aprendidas.

Es así como, el intercambio y socialización de las experiencias inició en los talleres realizados en las regiones como una de las actividades pre-foro. Allí, se identificaron y compartieron experiencias locales que fomentan la apropiación social de la CTeI, desde las necesidades propias de sus contextos. A partir de Este momento, se convocó e invitó a los líderes a presentar y registrar sus experiencias para el encuentro nacional.
El llamado a presentar experiencias en ASCTI iniciado en las regiones, se reforzó a través de la plataforma web del foro, Red Apropia, donde se dispuso un formulario para el registro de las experiencias que decidieran participar en el evento presencial, siendo la socialización de las mismas una de las actividades centrales del foro. En la plataforma se registraron 85 experiencias de todo el país, de las cuales fueron seleccionadas para presentarse en el encuentro 57.

Durante el foro, se presentaron alrededor de 70 experiencias en ASCTI, nacionales $\mathrm{E}$ internacionales. En la modalidad de ponencia, se presentaron 36 experiencias nacionales y 7 internacionales (Chile, España, Uruguay, Ecuador, Perú, Costa Rica y México) ${ }^{1} 18$ experiencias nacionales fueron presentadas en la modalidad de posters y 3 en el espacio de video-foro.

\footnotetext{
${ }^{1}$ Ver catálogo Experiencias Internacionales $\mathrm{CAB}$ http://convenioandresbello.org/inicio/
} 
Además, se contó con la presentación de programas y estrategias nacionales liderados por Colciencias y la Asociación Colombiana para el Avance de la Ciencia ACAC, como los son, Ideas para el Cambio, A ciencia cierta, Semana Nacional de la Ciencia, Programa Ondas y Encuentro con el futuro.

\section{¿DE DÓNDE Y QUIÉNES SE PRESENTARON?}

Las experiencias presentadas representaron diferentes zonas del país. Tuvieron presencia 18 departamentos (Norte de Santander, Santander, Cundinamarca, Meta, La Guajira, Valle del Cauca, Antioquia, Cauca, Bolívar, Nariño, Tolima, Arauca, Atlántico, Risaralda, Amazonas, Vaupés, Boyacá y Sucre). Se contó con una fuerte participación de los departamentos de Cundinamarca, Antioquia, Atlántico, Valle del Cauca y Santander.

En su mayoría, las experiencias son implementadas en localidades, comunas, municipios y veredas, ya que son procesos que se están adelantando en comunidades y organizaciones de base que lideran procesos sociales, según las necesidades e intereses particulares de los territorios.

Durante el encuentro, los líderes de las experiencias, además de compartir las actividades que desarrollan en Ciencia y Tecnología desde sus regiones, visibilizaron los intereses por ciertas temáticas que responden a sus contextos particulares. Por ejemplo, en las zonas de Santander, Cundinamarca y Tolima se están desarrollando procesos de apropiación social, especialmente de transferencia de tecnología, para mejorar los procesos agrícolas y agropecuarios.

En los departamentos de La Guajira, Bolívar y Atlántico, las experiencias están relacionadas con temas de protección y cuidado ambiental, sobre todo con el manejo del agua y los recursos naturales. Se encuentran experiencias como, Plan de Apoyo en el saneamiento básico para el manejo del agua potable en la vereda Leticia/Bolívar de la Universidad de San Buenaventura.

En las zonas de la Amazonía, Valle del Cauca, Boyacá y Cauca, los procesos de apropiación social de la CTeI, se están generando a partir de la necesidad de reconstruir culturalmente los territorios. Temas de memoria histórica, arte, saberes ancestrales, folclor y música, entre otros, son visibles en las experiencias presentadas.

En este sentido, el Foro logra mostrar, a partir de las experiencias, el panorama nacional, regional y local de la apropiación social de la Ciencia, la Tecnología y la Innovación, a partir de procesos cotidianos liderados en los territorios y de las comprensiones locales de la política de ASCTI vigente en el país, y cómo los contextos resaltan necesidades y temas específicos.

\section{¿QUIÉNES PARTICIPARON?}

Con la misma diversidad de los territorios, los actores participantes se hicieron visibles. En su mayoría, las experiencias presentadas durante el encuentro son lideradas o apoyadas por universidades y grupos de investigación de todo el país.

Se hicieron presentes representantes de las Universidades de Pamplona, Konrand Lorenz, La Guajira, Antioquia, San Buenaventura, Cooperativa, Libre, Área Andina, EAFIT, Norte, Santo Tomás y Universidad Pontificia Bolivariana. También se contó con la participación del SENA, con sus regionales y Establecimientos Educativos de Básica.

Participaron entidades como Fundación Social, Servicio Geológico Colombiano, Parque Explora, Observatorio Colombiano de Ciencia y Tecnología OCyT, Instituto de Investigación de Recursos Biológicos Alexander Von Humboldt (IAvH), Corporación Memoria y Saber Popular, Parque Ecológico Mundo Amazónico, Instituto Amazónico de Investigaciones Científicas SINCHI-Vaupés, Fundación Botánica, Zoológico de Barranquilla, Apropesca, entre otros.

Líderes comunitarios, investigadores, docentes y estudiantes fueron los encargados durante los tres días de encuentro, de socializar y compartir los procesos y las actividades de CTeI realizadas en todas las zonas del país, a través de alianzas y convenios entre diferentes entidades. El Foro se convirtió en un espacio para el reconocimiento de los trabajos que se están desarrollando en los territorios, además de generar la posibilidad de conectarse con otros, gestión algún tipo de alianzas e iniciar una red de apropiación social del conocimiento. 
Durante todo el encuentro, se contó con la participación de aproximadamente 300 asistentes, que aportaron en la discusión de las mesas de trabajo y asistieron a la presentación de las experiencias de ASCTI seleccionadas en todas las modalidades.

\section{NECESIDADES ENCONTRADAS}

Como se ha mencionado y se presentó en el encuentro nacional, las experiencias en ASCTI surgen de la identificación de necesidades particulares de los contextos y territorios. Es así como las experiencias buscan generar condiciones, estrategias, ambientes y prácticas que fomenten la apropiación social de la CTeI.

Experiencias como Inclusión de $I+D+i$ en la formación profesional del Centro Agropecuario La Granja Espinal/Tolima, Formación en Tecnología Educativa con docentes de La Guajira - Maicao/La Guajira y Universidad de los Niños - Medellín/Antioquia, son algunos de los procesos que surgen a partir de la identificación de la necesidad de formación y capacitación en comunidades específicas. Formación en uso de TIC, en uso de herramientas, en gestión y planeación, son algunos de los temas.

Relacionado a la necesidad de formación, algunas experiencias buscan fomentar prácticas de investigación desde diferentes actividades. Ciencia para la gente - Cundinamarca y la Investigación "Ciclo biológico de mariposas" - Villavicencio/Meta, estimulan prácticas de investigación con ciudadanos y estudiantes de básica respectivamente.

Otras experiencias se consolidan para mejorar las condiciones de vida de comunidades y de la población de ciertas zonas del país, generando procesos de apropiación social para el cuidado y preservación del medio ambiente y el manejo de los recursos naturales. Tales experiencias fueron presentadas en el foro como procesos dinámicos y activos, ya que los ciudadanos hacen posible su sostenibilidad. Entre algunas de estas se encuentra, Abastecimiento de agua a través del bombeo solar en la comunidad indígena Etkojo Manaure/La Guajira y Parque Mundo Amazónico Leticia/Amazonas.

Algunas experiencias, además de generar ciertas condiciones para mejorar aspectos específicos en los territorios, surgen porque identificaron dinámicas de exclusión e inequidad en las comunidades, relacionadas con uso y acceso a nuevas tecnologías, formación, acceso a recursos y espacios públicos. Ejemplos de estas son, Con la ciencia en la cabeza, el Radiality, que busca generar espacios de participación para los jóvenes de Medellín y Bosachoque - Libre, testimonio de inclusión social mediante el uso de redes libres, que busca generar acceso a internet a una población rural en Fusagasugá/Cundinamarca.

Otro importante grupo de experiencias responden a convocatorias específicas realizadas por Colciencias para generar procesos de apropiación social en los territorios. Estas experiencias fueron implementadas en diferentes lugares del país con metodologías diversas para acerca a los ciudadanos a las actividades de CTeI y generar apropiación social del conocimiento. Se destacan experiencias como: Metodología para la ASC generada por el proceso de planeación del desarrollo local en las Comunas $1 y 8$ de Medellín, Apropiación Social del Conocimiento en Salud en Santander y Conexiones entre museos y colegios: construcción colectiva de una metodología para la apropiación social del conocimiento en Barranquilla.

Entre otros temas, se encuentran la generación de alianzas, la gestión del conocimiento y la consolidación de espacios de diálogo entre investigadores-academia, líderes y ciudadanía acerca de temas relacionados con la CTeI.

\section{METODOLOGÍAS Y MATERIALES}

La generalidad en las metodologías desarrolladas es la participación activa de las comunidades y actores donde se implementan los procesos. Como lo plantean las mismas experiencias, las metodologías utilizadas fomentan la construcción colectiva, el diálogo, el intercambio de saberes y la producción de contenidos, incluso en los procesos de formación y de transferencia de conocimiento.

Las principales actividades desarrolladas que responden a los objetivos de las experiencias son los talleres de formación y las reuniones de socialización de las propuestas y de las acciones a realizar, ya que la mayoría de procesos son consultados y participan todos los actores. Estas actividades, en tal caso, son acompañadas por los semilleros de investigación. 
Las charlas, las salidas de campo y en general los encuentros son actividades que desarrollan la mayoría de las experiencias, ya que las metodologías dispuestas requieren de la construcción colectiva y de la generación de diálogos y reflexiones acerca de la apropiación social del conocimiento durante el proceso. Esto también les ha permitido conformar redes de trabajo y algunas alianzas.

Como parte del trabajo conjunto, se encuentra la realización de campañas comunicativas, la realización de eventos de divulgación, reuniones de socialización, uso de plataformas virtuales (portales, páginas Web, blogs) para generar constantemente información de la experiencia, intercambiar datos con otros y publicar los materiales o productos que se realizan. Un alto porcentaje de las experiencias recurren a medios electrónicos para mantener conexión con todos los participantes y dar a conocer su proceso.

En cuanto a los contenidos, materiales y productos que las experiencias realizan se encuentran, en su mayoría, documentos como guías de trabajo, guías con los contenidos de formación, folletos, juegos didácticos y manuales. En su mayoría, las experiencias comparten y publican sus materiales en sus plataformas virtuales o redes sociales; son contenidos que están visibles al público, pero que no han sido movilizados en otros espacios o escenarios distintos a las reuniones o encuentros de socialización que organizan, no siendo muy masivos.

Las experiencias que son acompañadas por grupos de investigación o están siendo desarrolladas por universidades cuentan con el desarrollo de artículos académicos, muchos de estos publicados. Son experiencias que ya han sido sistematizadas, que tienen mayor tiempo en la implementación y que, además, son parte de alianzas y convenios entre entidades, lo que facilita la sistematización y publicación de resultados.

A parte de las experiencias que se presentaron en la modalidad de "Video foro", Juego de héroes de Pampayan: historias de un reino olvidado, Popayán/ Cauca, Apropiación Social en la Provincia de Ocaña de Norte de Santander y Sistema de información para el acueducto San Roque, de Morales Cauca, otras han producido en su proceso contenidos audiovisuales o radiales, de los que se han encargado los mismos participantes, desde la construcción del guión hasta la post-producción.

Entre otros materiales producidos, se encuentra la construcción de encuestas, formatos de evaluación, bases de datos y formatos para la documentación de las actividades realizadas.

\section{AVANCES SIGNIFICATIVOS}

En el encuentro, las experiencias presentaron avances significativos, según el tiempo de implementación de las mismas. En todo caso, el desarrollo de materiales y productos, la socialización del proceso, la generación de alianzas o intercambio con otros y sobre todo la generación de apropiación social desde una comprensión local, son los factores que más se resaltan como logros y objetivos alcanzados.

Entre los avances que presentan las experiencias, se destacan los siguientes:

- Las experiencias que son implementadas por grupos de investigación señalan que un avance importante es la vinculación de más actores al grupo. Así mismo, el fortalecimiento de los semilleros de investigación son logros que se resaltan.

- La producción de artículos, contenidos audiovisuales, impresos, recursos multimediales, para la divulgación, comunicación y socialización de los procesos.

- Participación en ferias, eventos, congresos, foros, para compartir la experiencia. En muchos casos la participación fue para presentar ponencias y los productos desarrollados.

- Obtención de reconocimientos por otras entidades. Participación en convocatorias o concursos como ferias de ciencia y tecnología. En general, la oportunidad de socializar los procesos en otros escenarios es un logro significativo.

- Diseño de nuevas estrategias y actividades para el fortalecimiento de las experiencias, según el seguimiento que se realiza a la implementación.

- Generación de mayor información de los procesos que se adelantan con la implementación de las experiencias, mayor divulgación y comunicación a otros. Mejora de 
los canales y medios de comunicación. Mayor uso de redes sociales y plataformas virtuales para publicación de información.

- Vinculación de participantes en las actividades desarrolladas. En muchas experiencias, su implementación les permitió ampliar la cobertura y el impacto, pero también la vinculación de nuevos actores, generando alianzas.

- Documentar los procesos, consolidación de archivos y sistematización de los procesos, es un avance que rescatan mucho los líderes, ya que esto permite la continuidad y evaluación de los procesos.

- Agendar temas de CTeI en la región y en los escenarios locales como un aspecto necesario para el desarrollo social, cultural y económico de las regiones.

- Generación de comprensiones alrededor de la apropiación social de la CTeI en los contextos locales y nacionales.

- Aumento del interés de la comunidad académica, entidades del estado y ciudadanía en los procesos de CTeI que se desarrollan en los territorios y en el país a partir de la política de ASCTI.

- Fomento de metodologías, estrategias, planes, espacios para la Apropiación Social de la Ciencia, la Tecnología y la Innovación.

\section{OPORTUNIDADES}

Las oportunidades de mejoramiento identificadas por las experiencias tienen mucha relación con los avances que han logrado y son el resultado de los procesos de evaluación y seguimiento que adelantan las iniciativas.

Generar mayores intercambios entre investigadores, academia y la ciudadanía, en relación con procesos de CTeI, es uno de los grandes retos que presentan las experiencias. Afirman que la articulación debe darse de manera permanente, pero aún existen sesgos para compartir los saberes que se construyen en cada ámbito. Se afirma que la academia necesita acercarse más a las comunidades y comunicar de manera más cercana los resultados de sus investigaciones, teniendo en cuenta los contextos y las necesidades locales. La ciudadanía, por su parte, debe vincularse de manera más activa a los procesos relacionados con ciencia y la tecnología que se adelantan en sus territorios.

Sin duda, estos acercamientos permiten mayores oportunidades de participación de actores y entidades en proyectos, programas, planes de CTeI y amplían la cobertura y la generación de alianzas con otros sectores. De esta manera se enriquecen las comprensiones y «maneras» de fomentar la apropiación social de la CTeI.

Así mismo, la ampliación en recursos, infraestructura, medios y herramientas para fortalecer los procesos es un aspecto que las experiencias buscan fortalecer en la medida en que se van desarrollando las actividades y se vinculan otros actores, esto permite la sostenibilidad y continuidad de los procesos, que es una de las preocupaciones manifestadas por algunos líderes; se requiere dejar capacidad instalada en las comunidades, formar lideres, replicar los procesos para que continúen, se fortalezcan y generen mayores impactos.

Para el tema de la sostenibilidad, como una oportunidad de mejoramiento, las experiencias proponen mantener vigentes las redes o comunidades de aprendizaje que logran formarse durante la implementación de las experiencias, además de la actualización y permanencia de las plataformas virtuales, producto de las iniciativas como estrategia de continuidad.

\section{¿DE QUÉ MANERA LAS EXPERIENCIA DESARROLLAN PROCESOS DE APROPIACIÓN SOCIAL DE LA CIENCIA, LA TECNOLOGÍA Y LA INNOVACIÓN?}

Las experiencias presentadas en el Foro representan diversas maneras de visibilizar la apropiación social del conocimiento y de comprender cómo se generan estos procesos en las regiones y cómo son reconocidos en la Política de ASCTI. Todas las iniciativas reconocen la necesidad e importancia de generar este tipo de espacios para compartir esas compresiones y cualificar los avances en CTeI en el país.

Diversas son las experiencias, los actores, las actividades y materiales que desarrollan las iniciativas, pero se logran identificar, a través de los diálogos generados en el encuentro, puntos en común con relación a la apropiación social del conocimiento en temas de CTeI. 


\section{ALGUNAS APUESTAS MANIFESTADAS:}

- La ASCTI se está desarrollando a través del impulso de proyectos de investigación que responden a las necesidades y contextos. La articulación de la academia con las comunidades y organizaciones genera, sin duda, nuevas formas de apropiar el conocimiento, ya que se intercambian y construyen nuevos saberes.

- Influir en la toma de decisiones de política en $\mathrm{CTeI}$ es fundamental para mantener vigente los procesos de apropiación social. La participación en este tipo de espacios públicos democratiza las convocatorias, los presupuestos y los programas que planten las entidades del Estado.

- El fomento y generación de buenas prácticas de todo tipo, investigativas, ambientales, comunicativas, relacionales, sociales, culturales, aportan a la apropiación del conocimiento regional y local.

- Contribuir de manera permanente al fortalecimiento y empoderamiento de los actores, de los procesos y del mismo conocimiento, genera nuevas relaciones $\mathrm{y}$ articulaciones entre los saberes y las realidades que se buscan transformar.

- Desde la comprensión de la apropiación social como procesos interactivos, de construcción colectiva y participativa donde se reconozcan las formas de relación entre la ciencia y la sociedad y las iniciativas y experiencias que surjan de esto respondan de manera acertada a las situaciones de los contextos próximos.

- Se generan dinámicas de ASCTI cuando se propician diálogos entre diversas formas de conocimiento, academia, ciudadanía, entidades, gestores sociales entre otros.
Catálogo de experiencias en ASCTI: experiencias en apropiación social de la ciencia, la tecnología y la innovación 